\title{
Characteristic roots of a second order retarded functional dif- ferential equation via spectral-tau method
}

\author{
Habeeb Kareem Abdullah, Amal Khalaf Haydar*, Kawther Reyadh Obead \\ Department of Mathematics, Faculty of Education for Girls, University of Kufa, Najaf, Iraq.
}

\begin{abstract}
In this paper, we have found the solution of second-order delay differential equations of retarded type with multiple delays. As well as developing an approximation for finding characteristic roots for such delay differential equations via the method of spectral tau which depends on the basis mixed Fourier basis or shifted Chebyshev polynomials.
\end{abstract}

Keywords: Linear functional-differential equations, IBVPs for linear higher-order equations, spectral theory of functional-differential operators.

2010 MSC: 34K06, 34K08.

(C)2020 All rights reserved.

\section{Introduction}

Delay differential equations, were initially introduced as works in the eighteenth century, but a regular study of equations contain a deviating argument appeared only in the twentieth century in a relationship with the applied sciences. The differential equations with a deviating argument arise out of applications in various aspects of knowledge such as the problems related with combustion in the rocket motion, the control and optimization, the problem of long-range planning in the economics, the systems of selfoscillating, many problems in biology, and science and technology. At present, this theory is one of the most important and rapid growth in the branches of mathematics [7]. The modelling of many problems in terms of delay differential equations has been developed in a vast range, especially systems of electricity and mechanics and interest in DDEs have continued to increase because it has become very important in the area of biomedical modelling (physiological and hormonal control systems) [13]. During the little years ago, the topic of differential equations with deviating arguments has been expanded extensively. It has become an essential part of the works that are dealing with certain applications, for example, viscoelasticity, distributed networks, heat flow, the interaction of species, learning models, and physiology [3]. Many researchers worked on finding analytical solutions and others worked on finding numerical solutions with studying the stability of oscillation for delay differential equations $[1,2,6,9-12,14]$.

\footnotetext{
*Corresponding author

Email addresses: habeebk. abdullah@uokufa.edu.iq (Habeeb Kareem Abdullah), amalkh.hayder@uokufa.edu.iq (Amal Khalaf Haydar)
}

doi: $10.22436 /$ jnsa.013.03.03

Received: 2019-07-28 Revised: 2019-09-17 Accepted: 2019-10-29 
The study of the stability of delay differential equations where the delays are multiple has been concerned with converting the delay differential equation to a partial differential equation (PDE) of boundary conditions. Then, the PDE can be approximated via spatial discretization methods to an ordinary differential equation (ODE), i.e., DDE with initial conditions can be converted to a DDE with mixed conditions initial and boundary, i.e., (IBVP) for the advection equation [4, 8]. Vyasarayani et al. developed approximations to find the characteristic roots of delay differential equations of the first order via the spectral methods [15]. In the present paper, the spectral-tau approach is used for finding the solution of a retarded type delay differential equation of the second order with multiple delays and the characteristic roots of that delay differential equation are given.

Now, we shall consider an nth order retarded dynamical system when the time delay $\mathrm{T}$ is a constant, the governing equation can be written as follows

$$
\sum_{i=0}^{n} a_{i} \frac{d^{i}}{d t^{i}} y(t)+\sum_{i=0}^{m} b_{i} \frac{d^{i}}{d t^{i}} y(t-T)=0,
$$

such that $a_{i}, b_{i}$, are constants, $a_{n} \neq 0, n>m$ and $T>0$ is a nonnegative [9].

\section{Solving a retarded functional differential equation of second order via spectral-tau method}

We want to solve the second order delay differential equation with delays $\tau_{1}, \tau_{2}, \cdots, \tau_{m}$

$$
\ddot{y}(t)+b \dot{y}(t)+\sum_{j=1}^{m} b_{j} \dot{y}\left(t-\tau_{j}\right)=0, \quad \tau_{j}>0,
$$

with the initial delay condition:

$$
y(t)=\phi(t), \quad-\tau \leqslant t \leqslant 0,
$$

where $\tau=\max \left\{\tau_{1}, \tau_{2}, \cdots, \tau_{m}\right\}$. By using the known shift time [15]

$$
u(p, t)=y(t+p), \quad p \in[-\tau, 0] .
$$

The IVP (2.1)-(2.2) can be expressed by an IBVP of the form

$$
\begin{gathered}
\frac{\partial^{2} u(p, t)}{\partial t^{2}}=\frac{\partial^{2} u(p, t)}{\partial p \partial t}, \quad-\tau \leqslant p \leqslant 0, \\
\left.\frac{\partial^{2} u(p, t)}{\partial t^{2}}\right|_{p=0}=-b \frac{\partial u(0, t)}{\partial t}-\sum_{j=1}^{m} b_{j} \frac{\partial u\left(-\tau_{j}, t\right)}{\partial t}, \\
u(p, 0)=\varphi(p), \quad-\tau \leqslant p \leqslant 0 .
\end{gathered}
$$

Also, using the spectral-tau approach, we suppose that there is a solution for the partial differential equation (2.3) can be in the following formula

$$
u(p, t)=\sum_{i=1}^{\infty} \alpha_{i}(p) \delta_{i}(t),
$$

such that $\alpha_{i}(p)$ will be known functions which are called basis functions and $\delta_{\mathfrak{i}}(t)$ are unknown functions which are called time dependent coordinates. For workable causes, we take the above summation at $\mathrm{N}$ of the terms as follows

$$
u(p, t)=\alpha(p)^{\top} \delta(t)
$$


such that

$$
\alpha(p)=\left[\alpha_{1}(p), \alpha_{2}(p), \alpha_{3}(p), \cdots, \alpha_{N}(p)\right]^{\top} \quad \text { and } \quad \delta(t)=\left[\delta_{1}(t), \delta_{2}(t), \delta_{3}(t), \cdots, \delta_{N}(t)\right]^{\top} .
$$

If we substitute the above series equation in (2.3) gives

$$
\alpha(p)^{\top} \ddot{\delta}(t)=\alpha^{\prime}(p)^{\top} \dot{\delta}(t) .
$$

By multiplying both sides with $\alpha(p)$, then integrating with respect to the variable $p$ from $-\tau$ to 0 yields

$$
\int_{-\tau}^{0} \alpha(p) \alpha(p)^{\top} d p \ddot{\delta}(t)=\int_{-\tau}^{0} \alpha(p) \alpha^{\prime}(p)^{\top} d p \dot{\delta}(t) .
$$

Equation (2.7) becomes

$$
\mathrm{G} \ddot{\delta}(\mathrm{t})=\mathrm{H} \dot{\delta}(\mathrm{t})
$$

where

$$
\begin{aligned}
& G=\int_{-\tau}^{0} \alpha(p) \alpha(p)^{\top} d p \\
& H=\int_{-\tau}^{0} \alpha(p) \alpha^{\prime}(p)^{\top} d p .
\end{aligned}
$$

Substituting (2.6) in (2.4) gives the scalar equation

$$
\alpha(0)^{\top} \ddot{\delta}(t)=\left[-b \alpha(0)^{\top}-\sum_{j=1}^{m} b_{j} \alpha\left(-\tau_{j}\right)^{\top}\right] \dot{\delta}(t) .
$$

It is clear that the two equations (2.8) and (2.9) produce $\mathrm{N}+1$ equations. To get a solvable system we respect the last equation of the system (2.8) and add (2.9) to construct

$$
\mathrm{U}_{\mathrm{tau}} \ddot{\delta}(\mathrm{t})=\mathrm{V}_{\mathrm{tau}} \dot{\delta}(\mathrm{t}),
$$

where

$$
\begin{aligned}
& \mathrm{u}_{\mathrm{tau}}=\left[\begin{array}{c}
\overline{\mathrm{G}} \\
\alpha(0)^{\mathrm{T}}
\end{array}\right] \\
& v_{\mathrm{tau}}=\left[\begin{array}{c}
\overline{\mathrm{H}} \\
-\mathrm{b} \alpha(0)^{\mathrm{T}}-\sum_{j=1}^{\mathrm{m}} b_{j} \alpha\left(-\tau_{j}\right)^{\mathrm{T}}
\end{array}\right]
\end{aligned}
$$

and the matrices $\bar{G}$ and $\bar{H}$ yield by neglecting the $n$-th row of the matrix $G$ and the $n$-th row of the matrix $\mathrm{H}$ respectively.

Now, we reduce the order of $N \times N$ system (2.10) of second order ODEs to $2 N \times 2 N$ system of first order ODEs by the assumption

$$
\dot{\delta}(\mathrm{t})=\mathrm{Q}(\mathrm{t}),
$$

which gives the following $2 \mathrm{~N} \times 2 \mathrm{~N}$ system of ODEs of the first order

$$
\left[\begin{array}{c}
\dot{\delta}(t) \\
\dot{Q}(t)
\end{array}\right]=M\left[\begin{array}{l}
\delta(t) \\
Q(t)
\end{array}\right]
$$

where

$$
M=\left[\begin{array}{cc}
0 & \mathrm{I}_{\mathrm{N}} \\
0 & \mathrm{U}_{\mathrm{tau}}^{-1} \mathrm{~V}_{\text {tau }}^{-1}
\end{array}\right]
$$

such that 0 denotes the matrix with entires zeros, $I_{N}$ represents $\mathrm{N} \times \mathrm{N}$ identity matrix, $\mathrm{U}_{\mathrm{tau}}$ is a nonsin- 
gular matrix defined in (2.11) and $V_{t a u}$ is defined in (2.12). The initial condition for (2.10) is

$$
\delta(0)=\mathrm{U}_{\mathrm{tau}}^{-1} \int_{-\tau}^{0} \alpha(p) \varphi(p) d p .
$$

It is clear that the solution of (2.1) becomes

$$
y(t)=u(0, t)=\alpha(0)^{\top} \delta(t) .
$$

We note that the delay differential equation (2.1) has approximated to the system (2.10). Substituting $y(t)=e^{\lambda t}$ in (2.1) gives the characteristic equation for (2.1) as

$$
\mathrm{D}(\lambda)=\lambda+\mathrm{b}+\sum_{j=1}^{m} b e^{-\lambda \tau_{j}}=0
$$

The spectrum of (2.1) represents the roots of the characteristic equation, i.e.,

$$
S_{\text {exact }}=\left\{\lambda_{i} \mid D\left(\lambda_{i}\right)=0, \operatorname{Re}\left(\lambda_{1}\right) \geqslant \operatorname{Re}\left(\lambda_{2}\right) \geqslant \cdots\right\} .
$$

The spectrum of (2.1) which is obtained by finding the eigenvalues of the $2 \mathrm{~N} \times 2 \mathrm{~N}$ system equation (2.13) which is defined as

$$
\hat{S}_{\text {tau }}=\left\{\hat{\lambda}_{i}: \operatorname{det}(M-\hat{\lambda} I)=0, \quad \operatorname{Re}\left(\hat{\lambda}_{1}\right) \geqslant \operatorname{Re}\left(\hat{\lambda}_{2}\right) \geqslant \cdots\right\},
$$

where $M$ is $2 \mathrm{~N} \times 2 \mathrm{~N}$ matrix and defined as in (2.14).

As we know that choosing basis functions $\alpha(p)$ in (2.6) make a great effect in convergence or divergence of the eigenvalues. Therefore, we shall deal with the following basis functions: mixed Fourier basis [16]:

$$
\alpha(p)=\left[1, p, \sin \left(\frac{\pi}{\tau} p\right), \sin \left(2 \frac{\pi}{\tau} p\right), \cdots\right]^{\tau},
$$

and shifted Chebyshev polynomials [5]

$$
\alpha_{1}(p)=1, \quad \alpha_{2}(p)=1+\frac{2 p}{\tau}, \quad \alpha_{i}(p)=2 \alpha_{2}(p) \alpha_{i-1}(p)-\alpha_{i-2}(p), \quad i=3,4, \cdots .
$$

\section{Explained examples}

Example 3.1. Consider the following IVP of the second order

$$
\ddot{y}(t)+\dot{y}(t)+\dot{y}(t-5)=0,
$$

with the initial delay condition

$$
y(t)=\varphi(t), \quad-5 \leqslant t \leqslant 0 .
$$

We can find the exact characteristic roots of (3.1) using Lambert $W$ function as

$$
\lambda_{k}=\frac{1}{5} W_{k}\left(-5 e^{5}\right)-1, \quad k=0, \mp 1, \mp 2, \cdots,
$$

where $W_{k}, k=0, \mp 1, \mp 2, \cdots$ are branches of Lambert $W$ function.

Now, we consider that $u(p, t)=y(t+p), p \in[-5,0]$. The initial value problem (3.1)-(3.2) can be expressed by the following initial boundary value problem, by using the equations (2.3), (2.4), (2.5), as follows

$$
\begin{gathered}
\frac{\partial^{2} u(p, t)}{\partial t^{2}}=\frac{\partial^{2} u(p, t)}{\partial p \partial t}, \quad-5 \leqslant p \leqslant 0, \\
\left.\frac{\partial^{2} u(p, t)}{\partial t^{2}}\right|_{p=0}=-\frac{\partial u(0, t)}{\partial t}-\frac{\partial u(-5, t)}{\partial t}, \\
u(p, 0)=\varphi(p), \quad-5 \leqslant p \leqslant 0
\end{gathered}
$$

such that

$$
u(p, t)=\alpha(p)^{\top} \delta(t)
$$


where

$$
\alpha(p)=\left[\begin{array}{c}
\alpha_{1}(p) \\
\alpha_{2}(p) \\
\cdot \\
\cdot \\
\cdot \\
\alpha_{20}(p)
\end{array}\right], \quad \delta(t)=\left[\begin{array}{c}
\delta_{1}(t) \\
\delta_{2}(t) \\
\cdot \\
\cdot \\
\cdot \\
\delta_{20}(t)
\end{array}\right],
$$

and $\alpha(p)$ is either mixed Fourier basis or shifted Chebyshev polynomials which are defined as in (2.16) and (2.17) respectively.

Figure 1 shows the exact characteristic roots $S_{\text {tau }}$ of the DDE (3.1) computed form (3.3), and the approximated characteristic roots $\hat{S}_{\text {tau }}$ of the DDE (3.1) computed form (2.15) with respect to mixed Fourier basis and shifted Chebyshev polynomials when $\mathrm{N}=20$.

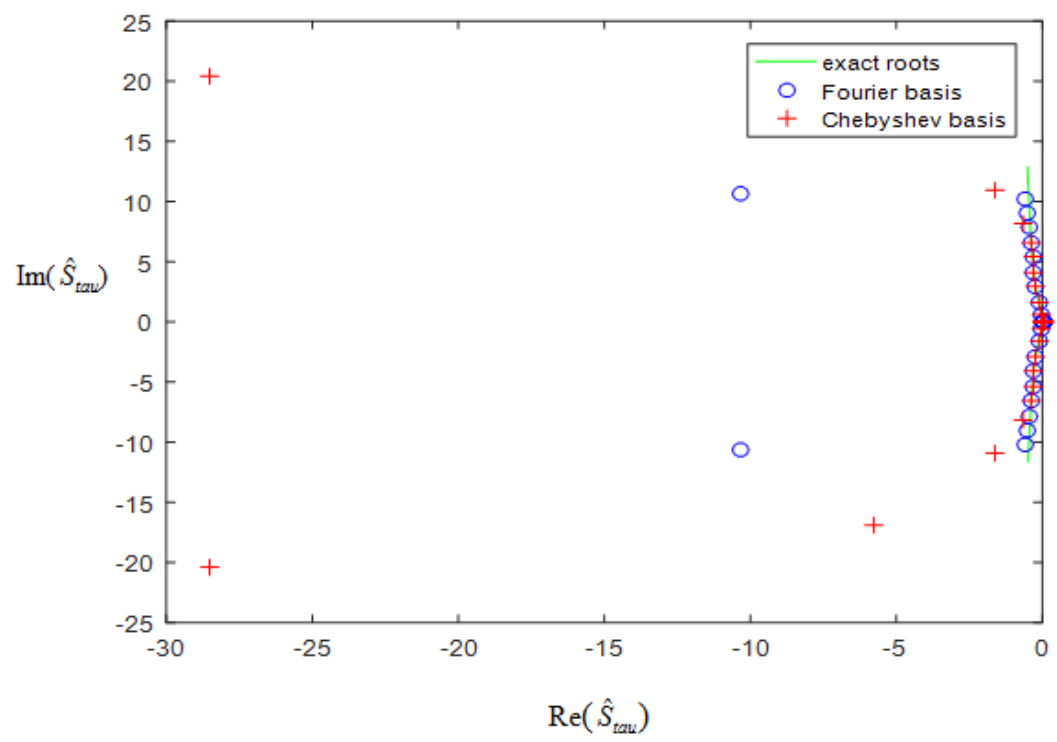

Figure 1: The exact $S_{\text {exact }}$ and approximated $S_{\text {tau }}$ characteristic roots of the DDE (3.1) when N=20.

Example 3.2. Consider the following IVP of the second order

$$
\begin{aligned}
\ddot{y}(t) & +3 \dot{y}(t)-2 \dot{y}(t-0.2)+\dot{y}(t-0.4)+2 \dot{y}(t-0.8)+2 \dot{y}(t-1)-\dot{y}(t-1.2) \\
- & \dot{y}(t-1.4)+3 \dot{y}(t-1.6)+\dot{y}(t-1.8)+\dot{y}(t-2)+\dot{y}(t-2.3)-\dot{y}(t-2.6) \\
& +\dot{y}(t-2.9)-\dot{y}(t-3)-5 \dot{y}(t-3.5)=0,
\end{aligned}
$$

with the initial delay condition

$$
y(t)=\varphi(t), \quad-3.5 \leqslant t \leqslant 0 .
$$

Now, we consider that $u(p, t)=y(t+p), p \in[-3.5,0]$.

Using (2.3), (2.4), (2.5), the initial value problem (3.4)-(3.5) can be expressed by an IBVP of the formulation

$$
\begin{aligned}
\frac{\partial^{2} u(p, t)}{\partial t^{2}}= & \frac{\partial^{2} u(p, t)}{\partial p \partial t}, \quad-3.5 \leqslant p \leqslant 0, \\
\left.\frac{\partial^{2} u(p, t)}{\partial t^{2}}\right|_{p=0}= & -3 \frac{\partial u(0, t)}{\partial t}+2 \frac{\partial u(-0.2, t)}{\partial t}-\frac{\partial u(-0.4, t)}{\partial t}-2 \frac{\partial u(-0.8, t)}{\partial t} \\
& -2 \frac{\partial u(-1, t)}{\partial t}+\frac{\partial u(-1.2, t)}{\partial t}+\frac{\partial u(-1.4, t)}{\partial t}-3 \frac{\partial u(-1.6, t)}{\partial t}-\frac{\partial u(-1.8, t)}{\partial t}
\end{aligned}
$$




$$
\begin{aligned}
& -\frac{\partial u(-2, t)}{\partial t}-\frac{\partial u(-2.3, t)}{\partial t}+\frac{\partial u(-2.6, t)}{\partial t}-\frac{\partial u(-2.9, t)}{\partial t}+\frac{\partial u(-3, t)}{\partial t} \\
& +5 \frac{\partial u(-3.5, t)}{\partial t}, \\
& u(p, 0)=\varphi(p), \quad-3.5 \leqslant p \leqslant 0,
\end{aligned}
$$

such that

$$
u(p, t)=\alpha(p)^{\top} \delta(t)
$$

where

$$
\alpha(p)=\left[\begin{array}{c}
\alpha_{1}(p) \\
\alpha_{2}(p) \\
\cdot \\
\cdot \\
\cdot \\
\alpha_{20}(p)
\end{array}\right], \quad \delta(t)=\left[\begin{array}{c}
\delta_{1}(t) \\
\delta_{2}(t) \\
\cdot \\
\cdot \\
\cdot \\
\delta_{20}(t)
\end{array}\right],
$$

and $\alpha(p)$ is either mixed Fourier basis or shifted Chebyshev basis which are defined as in (2.16) and (2.17) respectively.

Figure 2 shows the approximated characteristic roots $\hat{S}_{\text {tau }}$ of the DDE (3.4) computed form (2.15) with respect to mixed Fourier basis and shifted Chebyshev basis when $\mathrm{N}=20$.

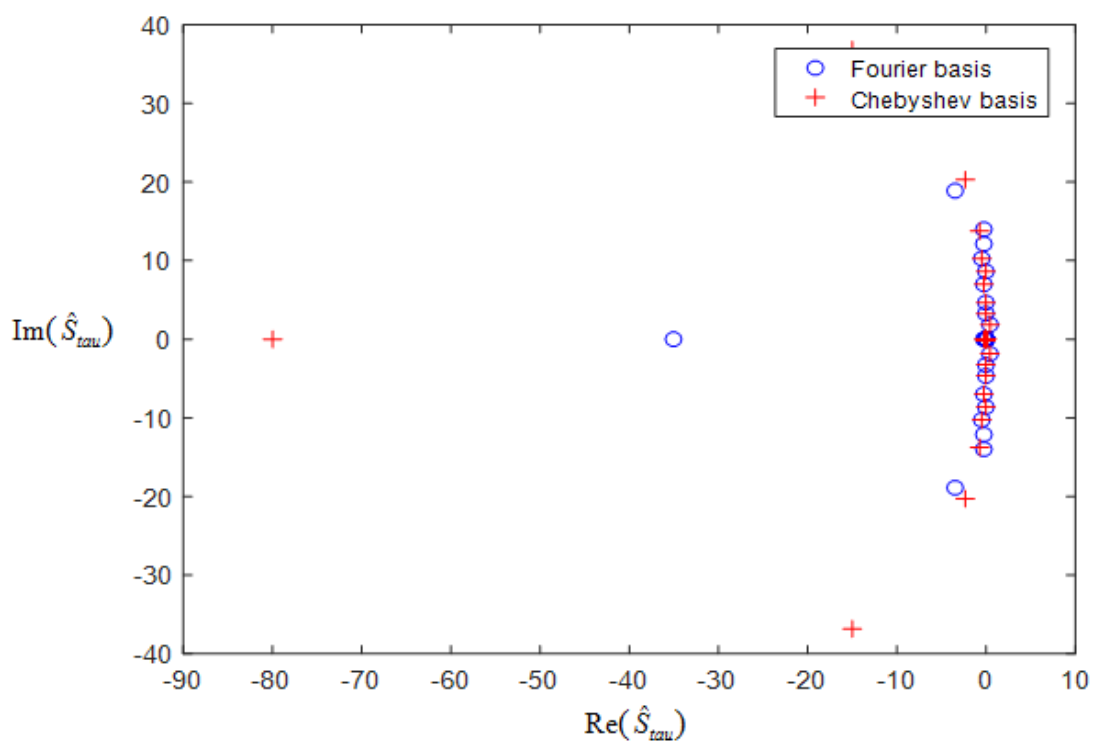

Figure 2: The approximated characteristic roots $\widehat{S}_{t a u}$ of the DDE (3.4) by mixed Fourier basis and shifted Chebyshev polynomials when $\mathrm{N}=20$.

\section{Conclusions}

We have introduced an approximation for the characteristic roots of a retarded functional differential equation with multiple delays when two basic functions that are: mixed Fourier basis and shifted Chebyshev polynomials. We noted that the choice of basis functions plays a substantial effect in convergence and divergence of the resulting eigenvalues because they effect on the singularity or non-singularity of the matrix $\mathrm{U}_{\mathrm{tau}}$. 


\section{References}

[1] H. K. Abdullah, A. K. Haydar, K. R. Obead, Solving of third order retarded dynamical system via lambert W function and stability analysis, World Wide J. Multidisciplin. Res. Develop., 4 (2018), 105-112. 1

[2] H. K. Abdullah, A. K. Haydar, K. R. Obead, Solving retarded dynamical system of nth-order and stability analysis via lambert W function, Int. J. Pure Appl. Math., 118 (2018), 2567-2584. 1

[3] O. Arino, M. L. Hbid, E. A. Dads, Delay differential equations and application, Springer, The Nethelands, (2006). 1

[4] A. Bellen, S. Maset, Numerical solution of constant coefficient linear delay differential equations as abstract Cauchy problems, Numer. Math., 84 (2000), 351-374. 1

[5] J. P. Boyd, Chebyshev and Fourier Spectral Methods, Dover Publ. Inc., Mineola, (2001). 2

[6] S.-T. Chen, S.-P. Hsu, H.-N. Huang, B.-Y. Yang, Time response of a scalar dynamical system with multiple delays via Lambert W functions, arXiv, 2016 (2016), 24 pages. 1

[7] L. E. Él'sgol'st, S. B. Norkin, Introduction to the theory and application of differential equations with deviating arguments, Academic Press, New York-London, (1973). 1

[8] T. Koto, Method of lines approximations of delay differential equations, Comput. Math. Appl., 48 (2004), 45-59. 1

[9] M. S. Lee, C. S. Hsu, On the $\tau$-decomposition method of stability analysis for retarded dynamical systems, SIAM J. Control, 7 (1969), 242-259. 1

[10] T. X. Li, B. Baculíková, J. Džurina, C. H. Zhang, Oscillation of fourth-order neutral differential equations with $p-$ Laplacian like operators, Bound. Value Probl., 2014 (2014), 9 pages.

[11] Q. Li, R. Wang, F. Chen, T. X. Li, Oscillation of second-order nonlinear delay differential equations with nonpositive neutral coefficients, Adv. Difference Equ., 2015 (2015), 7 pages.

[12] I. Mezo, G. Keady, Some physical applications of generalized Lambert functions, Eur. J. Phys., 37 (2016), 10 pages. 1

[13] K. Schmitt, Delay and functional differential equations and their applications, Academic Press, New York-London, (1972). 1

[14] S. S. Sujitha, D. Piriadarshani, A Lambert W function a approach for solution of second order delay differential equation as a special case of the one-mass system controlled over the network, Int. J. Mech. Eng. Tech., 8 (2017), 502-511. 1

[15] C. P. Vyasarayani, S. Subhash, T. Kalmar-Nagy, Spectral approximations for characteristic roots of delay differential equations, Int. J. Dynam. Control., 2 (2014), 126-132. 1, 2

[16] P. Wahi, A. Chatterjee, Galerkin projections for delay differential equations, J. Dyn. Syst. Meas. Control., 127 (2005), 80-87. 2 